\title{
IDENTIFICATION OF MYOCARDIAL INFARCTION USING THREE-DIMENSIONAL STRAIN TENSOR FRACTIONAL ANISOTROPY
}

\author{
Sahar Soleimanifard ${ }^{1}$, Khaled Z. Abd-Elmoniem ${ }^{1}$, Harsh K. Agarwal ${ }^{1}$, Miguel S. Tomas $^{2}$, Tetsuo Sasano ${ }^{2}$, \\ Evertjan Vonken ${ }^{2}$, Amr Youssef ${ }^{2}$, M. Roselle Abraham ${ }^{2}$, Theodore P. Abraham ${ }^{2}$, Jerry L. Prince ${ }^{1}$ \\ ${ }^{1}$ Department of Electrical and Computer Engineering, \\ ${ }^{2}$ Department of Cardiology, School of Medicine, \\ Johns Hopkins University, Baltimore, M.D., USA
}

\begin{abstract}
Accurate localization of myocardial viability is important in diagnosis of infarction. Regional strain function provides excessive information for clinical decision making but comparison of strain tensor profiles across differing tissue types is usually difficult due to multi-variate nature of tensors. It is desirable to describe tensors with simplified scalar indices which are more mathematically and statistically intuitive. In this work, anisotropy of tensors in healthy and experimental infarct regions in a large animal model is assessed and compared to directional components of strain tensors which are currently the most popular indices in active use. Myocardial strain tensors are computed using zHARP, a magnetic resonance (MR) tagging technique that provides quantification of cardiac function with direct computation of three-dimensional tensors from two-dimensional short axis MR images. Fractional anisotropy of strain tensors shows high correlation with late gadolinium enhanced images and is capable of discrimination between healthy and infarcted regions.
\end{abstract}

Index Terms - Strain tensor, HARP, zHARP, Fractional Anisotropy, Infarction

\section{INTRODUCTION}

Characterization of myocardial structure and function is a crucial issue in clinical diagnosis of cardiac disease. Many techniques such as HARP [1], DENSE [2], SENC [3] has been proposed to quantify cardiac motion and strain. Although three-dimensional (3D) strain tensor quantification is readily accomplished, providing extensive amount of information, the adoption of these information into clinical practice is rare due to the difficulty in visual and quantitative interpretation of 3D tensor components. It is desirable to represent tensors with simplified scalar indices. Conventional indices are derived from strain tensors on the basis of the orientation of myocardium. The circumferential strain which corresponds to short axis compression is parallel to epicardial surface and counterclockwise, as viewed from the base. The radial strain

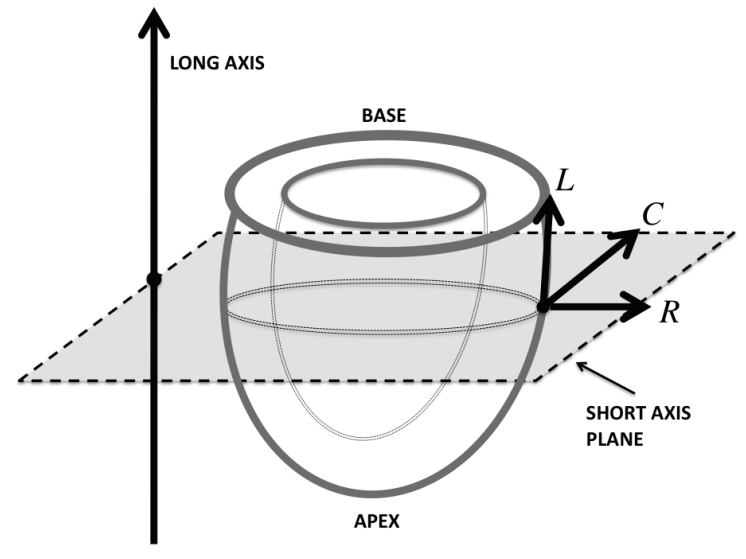

Fig. 1. Local coordinate systems. A separate cartesian system is defined for each material point, in which the axes are radial $R$, circumferential $C$, and longitudinal $L$.

which corresponds to elongation of tissue in short axis is perpendicular to epicardial surface. The longitudinal strain is in the long axis plane, tangent to the epicardial surface, and corresponds to compression of heart from base to apex. Figure 1 shows these directions in a local coordinate system. The normal range of strain patterns as a function of position in myocardium and of time during cardiac cycle has been previously studied [4] and provided a reference with which strains in abnormal hearts can be compared.

Tensors can also be described as ellipsoids consisting of three orthogonal vectors that each has a direction and an associated magnitude. It is hypothesized that infarcted tissue will neither compress nor elongate as mush as in healthy, active muscle, and therefore has less directional strain tensors. Anisotropy, the property of being directionally dependent, can transfer the information of 3D strain tensors into a scalar measure which is more informative and easier to interpret. Different anisotropy indices have been introduced but the most popular index in use is fractional anisotropy (FA), 
which was first proposed in continuum mechanics [5]. In medicine, it is mainly used in diffusion tensor imaging literature [6].

FA can be calculated from any imaging method that acquires 3D displacement information. In this work we use zHARP [7] technique, an extension to SF- CSPAMM [8], which directly provides both 3D strain tensor and displacement vectors at any arbitrary material point from twodimensional (2D) short axis MR images. Ability of FA to differentiate between normal and infarcted regions is studied and compared to other existing strain measures. Results show strong correlation with late gadolinium enhanced (LGE) images [9].

\section{THEORY}

\section{1. zHARP 3D displacement field}

zHARP pulse sequence applies an additional z-gradient of different polarities in slice select direction before regular $2 \mathrm{D}$ HARP tagging acquisition. Based on the fundamental fact that phase of harmonic images are material properties [1] and ability of zHARP tracking in through-plane direction [10], four complex phase images can be derived from two perpendicular tags:

$$
\begin{aligned}
& I_{v+}(\mathbf{p}, t)=c(\mathbf{p}, t) e^{j\left(+\omega\left(x-u_{x}\right)+\kappa z\right)} \\
& I_{v-}(\mathbf{p}, t)=c(\mathbf{p}, t) e^{j\left(-\omega\left(x-u_{x}\right)+\kappa z\right)} \\
& I_{h+}(\mathbf{p}, t)=c(\mathbf{p}, t) e^{j\left(+\omega\left(y-u_{y}\right)-\kappa z\right)} \\
& I_{h-}(\mathbf{p}, t)=c(\mathbf{p}, t) e^{j\left(-\omega\left(y-u_{y}\right)-\kappa z\right)}
\end{aligned}
$$

Where $\kappa$ is the z-encoding frequency. $\omega$ is the tagging frequency and locates spectral peaks in Fourier domain. $c$ contains spin density and artifacts caused by susceptibility and inhomogeneity. $(\mathbf{p}(x, y, z), t)=[\mathbf{x}, \mathbf{y}, \mathbf{z}]$ is the reference map of the material point which is at position $[x, y, z]$ at time $t$. $\left[u_{x}, u_{y}\right]$ shows displacement between reference frame and time $t$ :

$$
\begin{aligned}
& u_{x}=x-\mathbf{x} \\
& u_{y}=y-\mathbf{y}
\end{aligned}
$$

Filtering four spectral peaks yields:

$$
\begin{aligned}
& \phi_{v+}=\left(+\omega\left(-u_{x}\right)+\kappa z\right) \\
& \phi_{v-}=\left(-\omega\left(-u_{x}\right)+\kappa z\right) \\
& \phi_{h+}=\left(+\omega\left(-u_{y}\right)-\kappa z\right) \\
& \phi_{h-}=\left(-\omega\left(-u_{y}\right)-\kappa z\right)
\end{aligned}
$$

Considering the known location of slice at reference time frame $\mathbf{z}=z_{0}$, these four equations can be solved for displacement field:

$$
\begin{aligned}
& u_{x}=-\left(\phi_{v+}-\phi_{v-}\right) / 2 \omega \\
& u_{y}=-\left(\phi_{h+}-\phi_{h-}\right) / 2 \omega \\
& u_{z}=\left(\phi_{v+}+\phi_{v-}-\phi_{I+}-\phi_{I-}\right) / 4 \kappa-z_{0}
\end{aligned}
$$

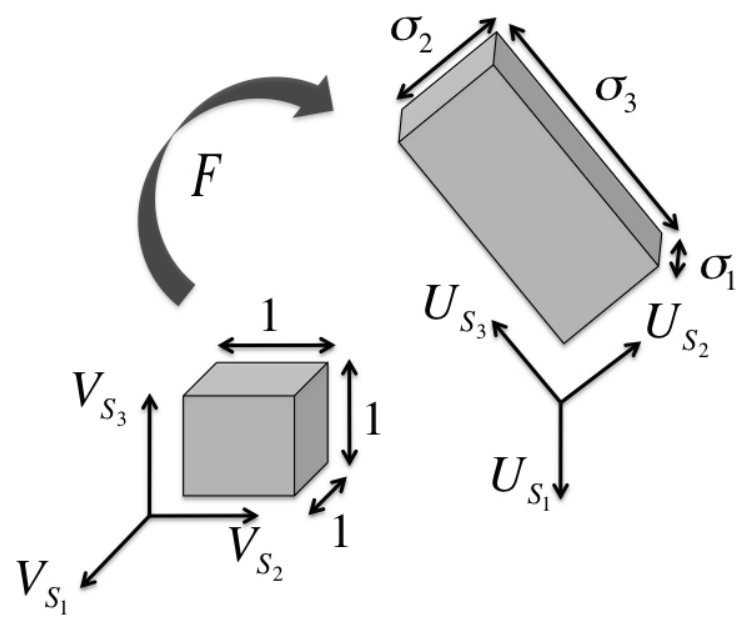

Fig. 2. Transformation of a unit cuboid in coordinate system $V_{s}$ into a deformed cuboid in coordinate system $U_{s}$. $U_{s}, V_{s}$, and $\sigma$ correpond to singular value decomposition of deformation gradient $F$.

By forming rectilinear mesh of points on a stack of equally separated images, displacement fields can be calculated for each spatial point during all cardiac phases. Deformation gradient and Eulerian strain can then be calculated at each point, yielding full strain tensor information in all myocardium.

\subsection{Deformation gradient and strain tensor}

Figure 2 shows the cuboid with three orthonormal vectors $V_{s}$ and edges of unit length, which undergoes deformation $F$, and reconfigures to the new cuboid with orthonormal vectors $U_{s}$ and edges of length $\left[\sigma_{1}, \sigma_{2}, \sigma_{3}\right]$. Singular value decomposition gives $F=U_{s} \Sigma_{s} V_{s}^{T}$ where $\Sigma_{s}=\operatorname{diag}\left(\sigma_{1}, \sigma_{2}, \sigma_{3}\right)$. Deformation gradient describes how an undeformed vector $d \mathbf{x}$ is transformed into $d x$. If the coordinate system at reference and later time is given by $\mathbf{x}$ and $x$ accordingly,

$$
\begin{aligned}
d x & =d u+d \mathbf{x} \\
F & =\frac{d x}{d \mathbf{x}}=I+\frac{d u}{d \mathbf{x}} \\
F^{-1} & =\frac{d \mathbf{x}}{d x}=I-\frac{d u}{d x}
\end{aligned}
$$

Where $u$ is displacement. $F$ enables us to estimate Eulerian finite strain tensor given by:

$$
e=\frac{1}{2}\left(I-F^{-T} F^{-1}\right)=\frac{1}{2} U_{s}\left(I-\Sigma_{s}^{-2}\right) U_{s}^{-T}
$$

Eigenvectors of $e$ are stored in columns of $U_{s}$, which correspond to principle or maximum directions of deformation. $\left(I-\Sigma_{s}^{-2}\right)$ is a diagonal matrix storing eigenvalues or magnitudes of deformation in principle directions. Tensor $e$ is rotated into local tissue coordinate system as shown in figure 1 

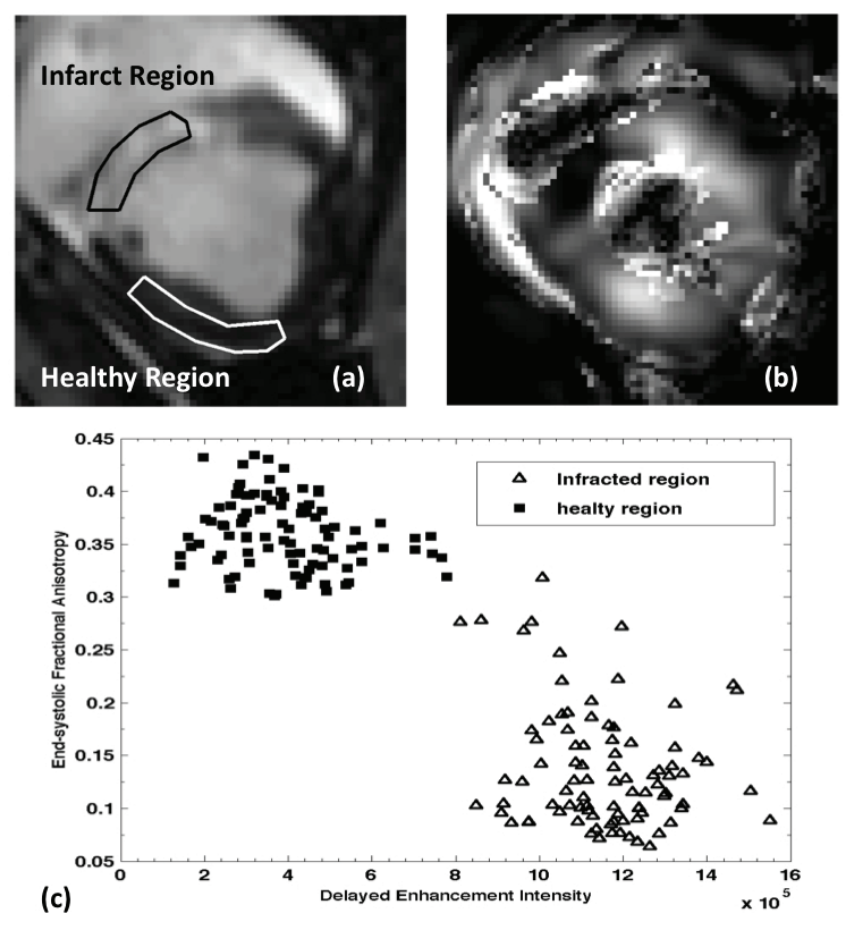

Fig. 3. (a) Delayed enhancement image at mid-diastole marked with infarcted and healthy tissue used for analysis. (b) Fractional anisotropy image at end-systole. (c) Plot of DE image intensity vs. FA taken from end-systole for healthy and infarcted regions shown in (a).

in order to compute directional strains. Using singular values of deformation gradient, FA can be calculated.

\subsection{Anisotropy of tensors}

Tensors can be described as isotropic or anisotropic. Isotropic tensors have the same components in all rotated coordinate systems and can be visualized as spheres with equal eigenvalues $\left(\lambda_{1}=\lambda_{2}=\lambda_{3}\right)$. In case of extreme anisotropic tensors eigenvalues are substantially different $\left(\lambda_{1}>\lambda_{2}>\lambda_{3}\right)$. FA $[5,6]$, a number between 0 and 1 , represents the degree of anisotropy, and is defined by:

$$
\begin{gathered}
\mathrm{FA}=\frac{\sqrt{3}}{\sqrt{2}} \frac{\sqrt{\left(\lambda_{1}-\lambda\right)^{2}+\left(\lambda_{2}-\lambda\right)^{2}+\left(\lambda_{3}-\lambda\right)^{2}}}{\sqrt{\lambda_{1}^{2}+\lambda_{2}^{2}+\lambda_{3}^{2}}} \\
\lambda=\left(\lambda_{1}+\lambda_{2}+\lambda_{3}\right) / 3 .
\end{gathered}
$$

\section{METHODS}

Myocardial infarction (MI) was induced by LAD coronary artery ligation in five pigs. Gadolinium contrast was performed 10-20 days after infarction and LGE viability images were acquired. The zHARP pulse sequence was run on a
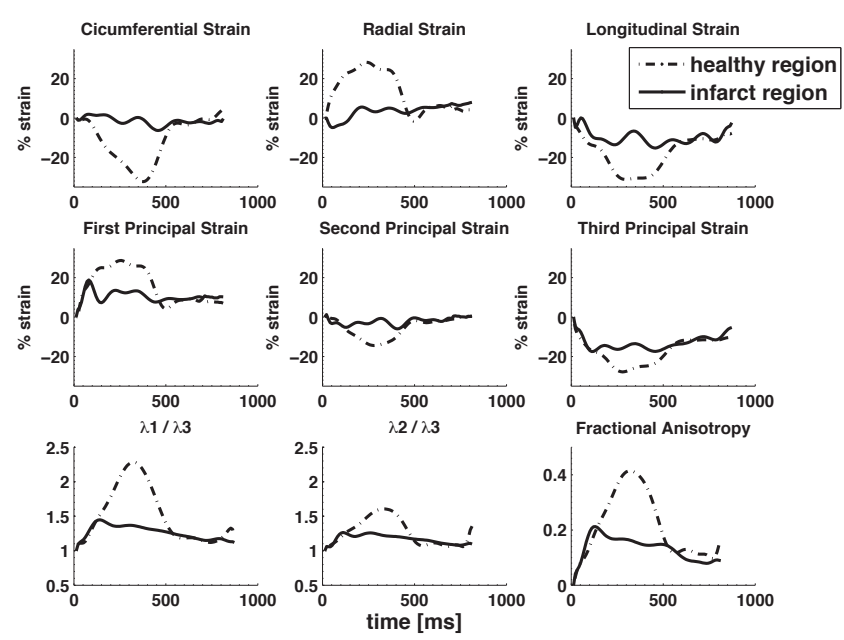

Fig. 4. Average value of strain indices within one cardiac cycle for regions marked in figure 3(a). Separation between two tissue types is evident.

commercial Philips 3.0T Achieva whole body MRI scanner (Philips Medical Systems, Best, NL), equipped with a sixchannel cardiac phased array surface coil. Channels were distributed equally between the anterior and posterior sides of the chest. 8-10 short axis slices from the base to apex were acquired using VECG triggered spiral imaging with a $9 \mathrm{~ms}$ acquisition window, 10 spiral readouts, 256 samples, FOV = $320 \mathrm{~mm}$, resolution $=1.25 \times 1.25 \mathrm{~mm}$, slice thickness $=8 \mathrm{~mm}$, $\mathrm{TR}=20 \mathrm{~ms}$, tag period $=7 \mathrm{~mm}$, and $\kappa=2 \pi / 33 \mathrm{rad} / \mathrm{mm}$. All the data were acquired using segmented k-space spiral acquisition.

\section{RESULTS AND DISCUSSION}

For each pig, mid-ventricular strain tensors were computed for all cardiac phases. Circumferential, radial, longitudinal strains, and FA was calculated for each animal. FA images were registered to the corresponding LGE images, and Harp tracking [10] was used to register the FA occurring at end systole with LGE intensities observed on the mid-diastolic geometry. Figure 3 (a) and (b) shows corresponding mid-diastolic LGE and end-systolic FA. Figure 3 (c) plots the intensity pairs (LGE,FA) for high-SNR healthy and infarcted regions with $r$ $=-0.89$ as shown in figure Fig 3 (a), where FA values are taken from end-systole.

Figure 4 shows mean value of strain indices within one cardiac cycle for both healthy and infarcted regions in one animal. Separation of these tissue types is evident in all data representations during systole when heart is compressed and tensors are anisotropic. Eigenvalues of $e$, form the principal values and correspond to two directions of maximum compression and one direction of maximum elongation in my- 


\begin{tabular}{|lllll|}
\hline & $\begin{array}{l}\text { Mean } \pm \text { std in } \\
\text { infarct region }\end{array}$ & $\begin{array}{l}\text { Mean } \pm \text { std in } \\
\text { healthy region }\end{array}$ & $\begin{array}{l}\text { Mean } \\
\text { CNR }\end{array}$ & $\begin{array}{l}\text { \%Cardiac cycle with } \\
\text { p<0.05 in two regions }\end{array}$ \\
\hline $\begin{array}{l}\text { \%Circumferential } \\
\text { Strain }\end{array}$ & $0.78 \pm 0.02$ & $-21.95 \pm 7.58$ & 3.30 & $66.66 \pm 3.16$ \\
\hline \%Radial Strain & $6.54 \pm 2.78$ & $20.02 \pm 6.77$ & 1.99 & $82.11 \pm 8.17$ \\
\hline \%Longitudinal Strain & $-7.12 \pm 3.41$ & $-29.11 \pm 13.02$ & 0.95 & $87.44 \pm 5.14$ \\
\hline \%First Principal Strain & $17.59 \pm 10.09$ & $39.73 \pm 14.69$ & 1.62 & $78.13 \pm 8.83$ \\
\hline $\begin{array}{l}\text { \%Second Principal } \\
\text { Strain }\end{array}$ & $0.94 \pm 1.42$ & $-7.95 \pm 6.9$ & 1.68 & $77.63 \pm 11.03$ \\
\hline $\begin{array}{l}\text { \%Third Principal } \\
\text { Strain }\end{array}$ & $-12.18 \pm 1.84$ & $-37.64 \pm 4.31$ & 2.73 & $89.20 \pm 4.22$ \\
\hline$\lambda_{1} / \lambda_{3}$ & $1.34 \pm 0.07$ & $2.19 \pm 0.15$ & 1.71 & $82.44 \pm 9.20$ \\
\hline$\lambda_{2} / \lambda_{3}$ & $1.15 \pm 0.02$ & $1.45 \pm 0.14$ & 1.10 & $86.76 \pm 10.94$ \\
\hline Fractional Anisotropy & $0.15 \pm 0.01$ & $0.41 \pm 0.07$ & 3.57 & $86.10 \pm 8.99$ \\
\hline
\end{tabular}

Table 1. Average end-systolic strain values, mean CNR and average $\%$ of cardiac cycle for $\mathrm{p}<0.05$ between two tissue types.

ocardium as expected. $\frac{\lambda_{1}}{\lambda_{3}}$ and $\frac{\lambda_{2}}{\lambda_{3}}$ confirms that tensors in region of infarction are substantially less anisotropic $\left(\lambda_{1} \approx\right.$ $\lambda_{2} \approx \lambda_{3}$ ). Tissue in this region is not elongated nor compressed in any directions and thus has a smaller FA compared to healthy region.

Marked regions of healthy and infarction are tracked in all cardiac phases for all five pigs. Table 1 lists average value of strain measures at end-systole when the heart is most compressed and corresponds to peak strain values. At this phase of cardiac cycle, contrast-to-noise-ratio (CNR) is calculated in both healthy and infarct regions and averaged over all pigs. Unpaired and unequal variance student $t$ test is performed on strains of both tissue types in one cardiac cycle. Mean percentage of cardiac cycle with $(\mathrm{p}<0.05)$ is reported. FA has less variance between subjects and has a relatively higher CNR. Circumferential strain shows a high CNR compared to others but shows discrimination for less fraction of cardiac cycle. Among the principle values, third value corresponds to maximum compression and has higher CNR than other two values. It also shows discrimination in larger fraction of cardiac cycle compared to others.

\section{CONCLUSION}

Spatially varying strain fields are mathematically more complex and computationally more intractable than are the data that compose scalar images. In this study we characterized regional cardiac strain in in-vivo subjects using FA. This anisotropy index shows high correlation with other conventional measures, and it has large CNR. FA encompasses the 3D information into a scalar which is independent of any particular direction and eliminates the need to screen all com- ponents of strain individually. In addition, FA is correlated with tissue viability and shows potential for localization of infarction without negative effects of contrast agent.

\section{REFERENCES}

[1] N.F. Osman, E.R. McVeigh, and J.L. Prince, "Imaging heart motion using harmonic phase mri," TMI, vol. 19, pp. 186-202, March 2000.

[2] A.H. Aletras, S. Ding, and R.S. Balaban, "Dense: displacement encoding with stimulated echoes in cardiac functional mri," J. Magn. Reson., vol. 137, pp. 247-52, March 1999.

[3] N.F. Osman, S. Sampath, and E. Atalar, "Imaging longitudinal cardiac strain on short-axis images using strainencoded mri," Magn. Reson. Med., vol. 46, pp. 324-34, August 2001.

[4] C.C. Moore, C.H. Lugo-Olivieri, E.R. McVeigh, and E.A. Zerhouni, "Three-dimensional systolic strain patterns in the normal human left ventricle: Characterization with tagged mr imaging," Radiology, vol. 214, pp. 453-66, February 2000.

[5] A.J.M. Spencer, Continuum Mechanics, Longman, London, 1980.

[6] P.J. Basser and C. Pierpaoli, "Microstructural and physiological features of tissues elucidated by quantitativediffusion-tensor mri," J. Magn. Reson., vol. 111, pp. 209-19, June 1996.

[7] K.Z. Abd-Elmoniem, M. Stuber, and J.L. Prince, "Direct three-dimensional myocardial strain tensor quantification and tracking using zharp," Medical Image Analysis, vol. 58, pp. 92-102, July 2007.

[8] S.E. Fischer, G.C. McKinnon, M.B. Scheidegger, W. Prins, D. Meier, and P. Boesiger, "True myocardial motion tracking," Magn. Reson. Med., vol. 12(6), pp. 778-86, December 2008.

[9] H. Mahrholdt, A. Wagner, R.M. Judd, U. Sechtem, and R.J. Kim, "Delayed enhancement cardiovascular magnetic resonance assessment of non-ischaemic cardiomyopathies," Eur. Heart J., vol. 26(15), pp. 1461-74, April 2005.

[10] K.Z. Abd-Elmoniem, N.F. Osman, and J.L. Prince, "Three-dimensional magnetic resonance myocardial motion tracking from a single image plane," Magn Reson Med, vol. 58, pp. 92-102, July 2007. 